Europhys. Lett., 72 (6), pp. 962-968 (2005)

DOI: $10.1209 / \mathrm{epl} / \mathrm{i} 2005-10325-6$

\title{
Coexistence of rhcp and fcc phases in hard-sphere colloidal crystals
}

\author{
I. P. Dolbnya ${ }^{1,2}\left(^{*}\right)$, A. V. Petukhov ${ }^{1}$, D. G. A. L. Aarts ${ }^{1}$, \\ G. J. VRoege ${ }^{1}$ and H. N. W. LEKKERKERKER ${ }^{1}$ \\ 1 Van 't Hoff Laboratory for Physical and Colloid Chemistry, Debye Institute \\ Utrecht University - Padualaan 8, 3584 CH Utrecht, The Netherlands \\ 2 DUBBLE CRG/ESRF, The Netherlands Organisation for Scientific Research \\ ESRF - BP 220, F-38043 Grenoble Cedex, France
}

received 18 March 2005; accepted in final form 13 October 2005

published online 11 November 2005

PACS. 61.10.-i - X-ray diffraction and scattering.

PACS. 82.70.Dd - Colloids.

PACS. 61.72.Nn - Stacking faults and other planar or extended defects.

\begin{abstract}
We present results for high-resolution small-angle single-crystal X-ray diffraction of hard-sphere silica colloidal crystals. Our results indicate that the stacking probability $\alpha$ of finding an fcc stacking sequence in a strongly compressed sample can only have two discrete values of either 0.5 (for rhcp) or 1 (for fcc) in contrast to previous observations on more dilute systems that metastable hard-sphere rhcp crystals may have various values of $\alpha$. This suggests that, in a concentrated sample, the transition from rhcp to fcc does not go by a smooth variation of $\alpha$, but through a rearrangement mechanism via melting, which leads to the formation of perfect fcc crystallites. These crystallites do not possess a single stacking fault as evidenced by the absence of twin fcc reflections.
\end{abstract}

Many monoatomic crystals with metallic or van der Waals bonding without pronounced directionality, display one of two closest packed structures, face centred cubic (fcc) or hexagonal close packed (hcp) with the $c / a$ ratio of the two basic lattice periods close to the "ideal" $c / a=\sqrt{8 / 3}$ value [1]. Both structures consist of hexagonal close packed layers, which are stacked with a lateral shift of one-third of the lattice period in the periodic ABCABC or $\mathrm{ABABAB}$ sequences, respectively, where $\mathrm{A}, \mathrm{B}$ and $\mathrm{C}$ denote the three possible lateral positions of the layers. The free energy difference between the fcc and hcp structures can be small, providing conditions to form extended two-dimensional defects by making errors in the stacking sequence. A well-known example is cobalt, which can form both, fcc $(\beta$-Co) and hcp $(\alpha-\mathrm{Co})$, structures. As much as $10 \%$ of stacking faults were seen in $\alpha$-Co [2-4].

A more recent example of close packed structures is provided by noninteracting colloidal hard spheres, which show a fascinating transition $[5,6]$ to a crystalline state. They serve as an important model system for various fields ranging from statistical mechanics through crystal

$\left(^{*}\right)$ Present address: Diamond Light Source, Rutherford Appleton Laboratory - Chilton, Didcot, Oxfordshire OX11 0QX, UK. E-mail: Igor.Dolbnya@diamond.ac.uk 
growth to soft matter physics and photonic engineering $[7,8]$. This self-ordering into crystalline structures at sufficiently high densities is somewhat counterintuitive since it is induced by entropy, which favours more effective packing and leaves more space for particle fluctuations around their equilibrium positions. Hard spheres possess no bond directionality and the free energies of the fcc and hcp crystal structures are nearly identical. Theory predicts [9] that for monodisperse spheres the fcc structure provides slightly larger space for particles fluctuations as compared to hcp, leading to a tiny free energy difference of only about $10^{-3} \mathrm{kT}$ per particle with $k T$ the thermal energy. Hard-sphere colloids are often observed [10-15] to have a randomly stacked hexagonal close packed (rhcp) structure, with practically equal probabilities of having stacking of the two types. Note that for small crystallites, which can act as nucleation centers, the additional contribution to the entropy originating from stacking disorder can stabilise the rhcp structure [16]. This situation is different from ordinary crystals, which choose for one of the two close packed crystal structure with, possibly, a small density of stacking faults.

Randomly stacked crystals possess a unique property, namely that interplanar periodicity is absent while long-range positional correlation persists over the whole crystal [14,15]. This interplanar positional order implies that any two atomic planes, even if they are far away from each other, are in one of the three possible distinct lateral positions (A, B or C). The stacking disorder leads to the appearance of so-called Bragg scattering rods in the reciprocal lattice of rhcp crystals in the direction normal to hexagonal layers. Periodicities common to all layers lead to sharp Bragg spots in which the diffracted intensity is localised in corresponding nodes of the crystal reciprocal lattice [4,17]. The stacking disorder is usually described by the probability $\alpha$ of finding an fcc stacking sequence among three randomly chosen subsequent layers.

As soon as the rhcp crystal is initially grown, there could then be three possible evolution scenarios. 1) The rhcp crystal structure can remain the stable phase over a long time. 2) The stacking faults can be "annealing" that will result in gradual structural changes of the rhcp crystals towards the slightly more energetically favourable fcc-type of lattice [13], thus leading to a continuous spectrum of the stacking parameter $\alpha$. 3) The rhcp crystal structure can be melted/dissolved out followed by the subsequent re-growth of fcc crystals $[18,19]$. The latter leads to a discrete spectrum of $\alpha$.

The stacking order can be directly probed by the confocal microscopy technique $[10,20,21]$. However, this can usually access only a limited number of crystal planes so that the estimate of the stacking probability $\alpha$ is statistically poor. Another approach is to explore diffraction techniques, using visible light or small-angle X-ray scattering, which provide information averaged over a macroscopic scale. Light scattering has been proven to be a powerful technique $[11-13,17,18]$ of structure evaluation of colloidal crystals. However, it can access only the low-order diffraction and significantly suffers from multiple scattering. Synchrotron X-ray sources allow to obtain clear diffraction patterns in a wide range of the scattering wave vectors $q$ and we here followed this approach.

The samples were prepared by sedimenting a dilute $5 \%$ volume fraction colloid suspension in a flat glass capillary of internal path length of $0.2 \mathrm{~mm}$. The silica spheres with diameter $D=224 \mathrm{~nm}$ and $4.1 \%$ size polydispersity were covered with a $2 \mathrm{~nm}$ thin hydrocarbon steric layer to prevent their agglomeration in the solvent cyclohexane [22]. The density contrast of the particles and the solvent is rather high, $\rho_{p}=1.69 \mathrm{~g} / \mathrm{ml}$ vs. $\rho_{s}=0.77 \mathrm{~g} / \mathrm{ml}$, respectively [23]. The potential energy of the spheres $m^{*} g h$ increases by $k T$ over the gravitational length $h=80 \mu \mathrm{m}$, where $m^{*}=\pi D^{3}\left(\rho_{p}-\rho_{s}\right) / 6$ is the buoyant mass, $g$ is the gravitational acceleration and $k T$ is the thermal energy. Osmotic compression by adding non-adsorbing polymer (polydimethylsiloxane with a molecular weight of $M_{w}=91.7 \mathrm{~kg} / \mathrm{mol}, 14 \mathrm{~nm}$ radius of gyration, at volume fractions of polymer coils of $10-15 \%$ around the phase separation 


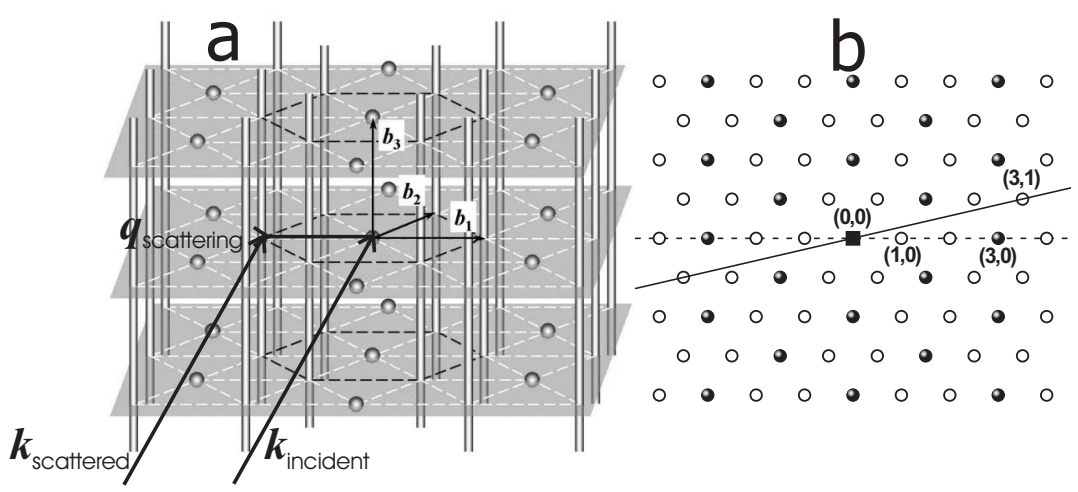

Fig. 1 - The scheme of the in-plane diffraction experiment. (a) Sketch of the reciprocal lattice of randomly stacked hexagonal close packed crystals. The shadowed planes are to guide the eye. (b) Top view of the reciprocal lattice illustrating the orientation of the Ewald sphere relative to the crystal reciprocal lattice. The closed symbols correspond to lines of localized reflections (spots) while open symbols display the position of rods.

boundary [23]) to the suspension is observed to accelerate crystallization and to give large high-quality crystals, which have been selected for the present study. The crystals have typically grown in the concentrated sediment within 1-2 weeks after sedimentation from the top of the sediment. The measured samples were about $1.5-2$ years old.

Small-angle X-ray diffraction (SAXD) measurements have been performed at the DutchBelgian bending magnet BM26B "DUBBLE" beam line at the European Synchrotron Radiation Facility (ESRF) in Grenoble (France) [24]. We have used a recently developed highresolution SAXD setup [25]. Monochromatic $15 \mathrm{keV}$ X-rays (wavelength $\lambda=0.83 \AA$, spectral width $\Delta \lambda / \lambda \approx 2 \times 10^{-4}$ ) were selected by a sagittally focusing (i.e., in the horizontal plane of the electron orbit) double-crystal $\mathrm{Si}(111)$ monochromator. It is followed by a bent silicon mirror, which focuses the beam in the vertical plane and discriminates against higher harmonics coming from the monochromator. The sample was mounted on computer-controlled rotation and translation stages allowing to scan through the sediment and to carefully orient the colloidal single crystals. The diffraction was recorded at a distance of $\sim 8 \mathrm{~m}$ from the sample by an X-ray sensitive 12-bit CCD (charge-coupled device) camera (X-Quis, Photonics Science). The diffraction images were digitized into $1024 \times 1024$ pixels with the pixel size corresponding to $\sim 52 \mu \mathrm{m}$ physical separation on the phosphor screen of the camera. The entire field of view was about $7.5 \mathrm{~cm}$ along the diagonal. To improve the angular resolution of the setup, the beam was carefully focused at the detector plane. The beam size at the sample position was about $150 \times 150 \mu \mathrm{m}^{2}$.

In the flat capillaries used one can find crystals with various orientations. Typically, individual crystallites are grown in a way that their hexagonal stacking layers are parallel to the capillary walls. However, in order to directly visualize the Bragg diffraction rods on the detector one needs to send the incoming wave vector, $\boldsymbol{k}_{i}$, parallel to hexagonal planes as is schematically outlined in fig. 1 . We were able to find crystals with hexagonal planes at an angle of about 60 degrees with respect to the capillary wall, thus enabling us to orient the sample in such a way that the incoming wave vector was parallel to the crystal planes. This produced diffraction patterns as shown in figs. 2a and b (patterns were taken at different points in the sample, thus originating from different single crystals). For these orientations, the Ewald sphere cuts through the reciprocal lattice as shown by the dashed and solid lines, respectively, in fig. $1 \mathrm{~b}$. 

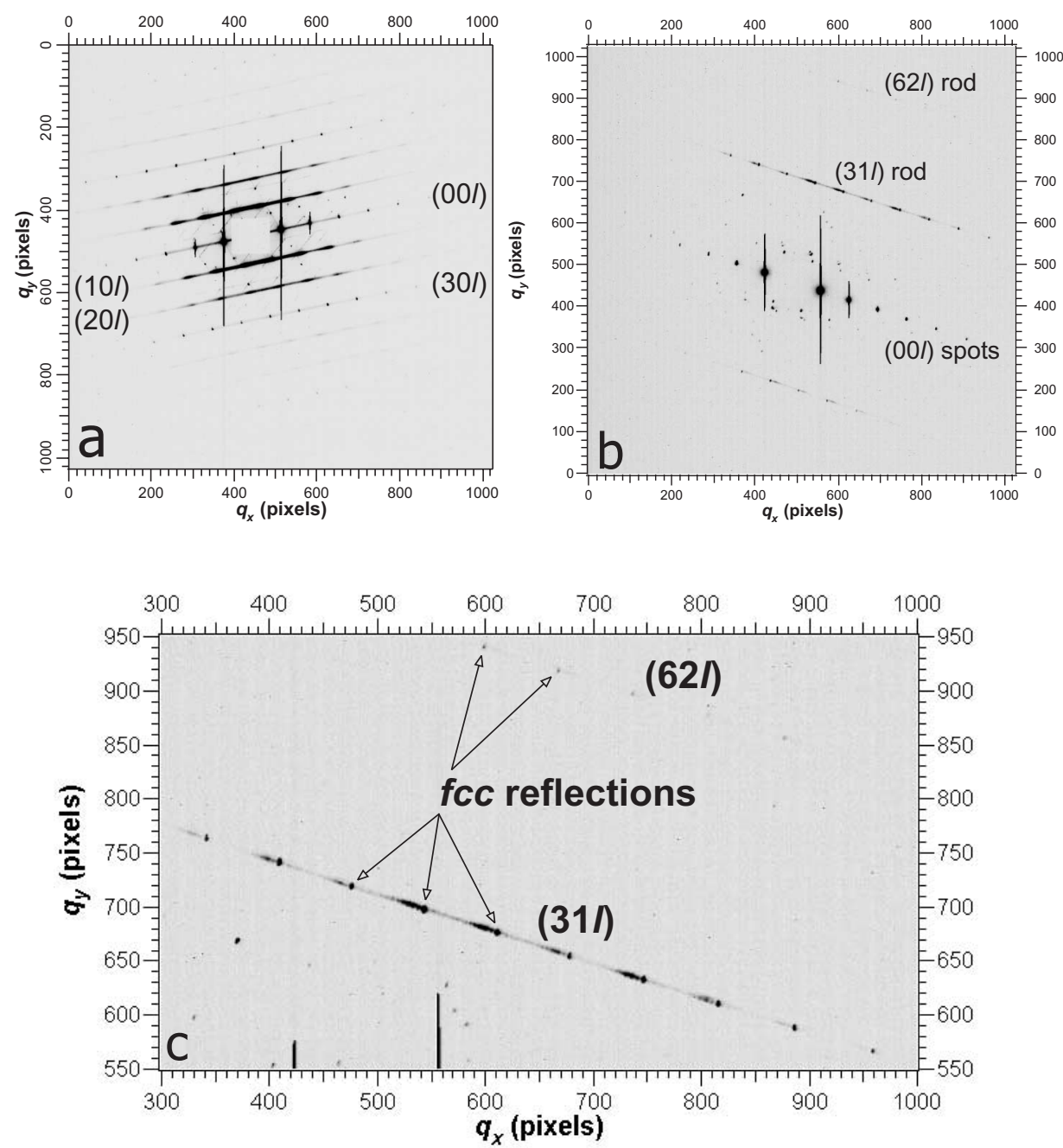

Fig. 2 - Diffraction patterns ("in-plane" diffraction geometry) measured for the crystal orientations corresponding to the Ewald sphere positions shown by dashed (a) and solid (b) lines in fig. 1(b). The direct beam is absorbed by a small beam stop in the middle of the detector. Panel (b) demonstrates the coexistence of rhcp and fcc crystals. (c) Magnified view of the scattering rods region of panel (b). The vertical stripes in the diffraction patterns are due to oversaturation of CCD camera used where the brightest reflections occur.

$\mathrm{X}$-ray scattering is observed on the detector along several lines, which originate from the Bragg rods and spots of the reciprocal lattice. The diffraction intensity along the Bragg rods smoothly varies and displays a periodic modulation with minima at integer values of $l$ and broad maxima in between them (fig. 2a). This profile of the structure factor along the rod is characteristic of an rhcp crystal with the stacking parameter $\alpha=0.5$ and is frequently observed in our samples. In some crystals, however, additional sharp reflections along the Bragg rods are observed, see fig. 2b. The improved instrumental resolution of the setup in $q$ space has made it possible to quantitatively resolve this in great detail. The reflections appear at $l=m-1 / 3$ for the $(31 l)$ rod, where $m$ is an integer (as they should occur for the Miller 


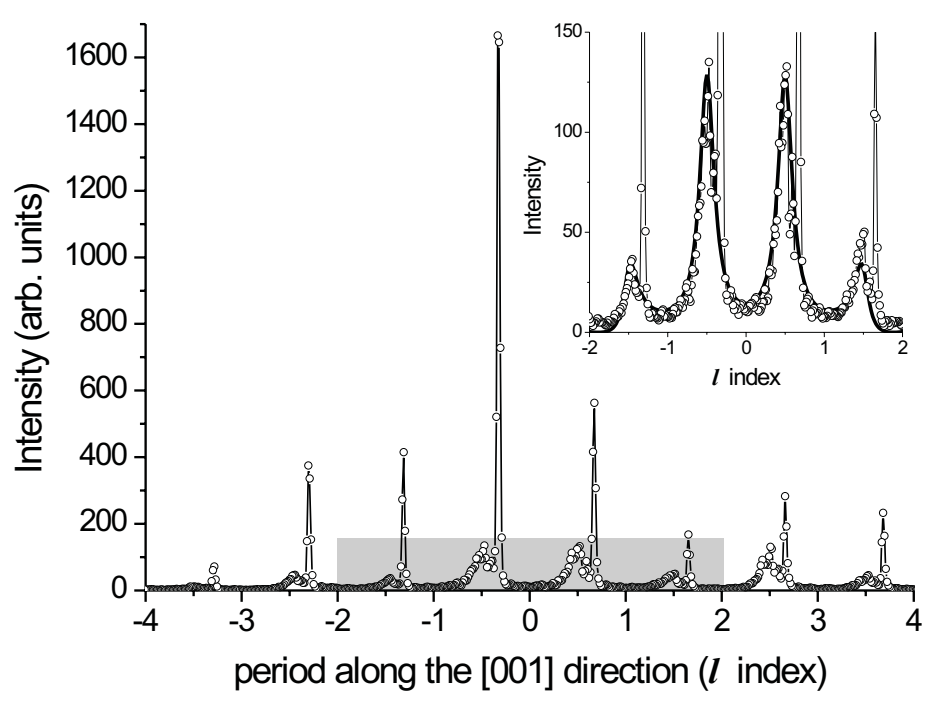

Fig. 3 - Coexistence of rhcp and fcc crystals: a slice of the diffracted intensity (averaged over the 10-pixel band) along the (31l) rod (see fig. $2 \mathrm{~b}, \mathrm{c}$ ). The background is subtracted. The inset presents a zoom of the gray-shaded area. The thick solid line is the result of a theoretical calculation with $\alpha=0.5$.

indices $h$ and $k$ satisfying the condition: $h-k=3 n+2, n$ integer). In the rod $(62 l)$, similar sharp reflections appear at $l=m+1 / 3$ (for $h-k=3 n+1$ ). The only plausible explanation for the existence of these sharp reflections is the presence of a single-domain perfect fcc crystal with one of its (111) planes attached to the hexagonal planes of the rhcp single crystal. Note that there are no twin fcc reflections in the diffraction patterns. This means that the fcc crystal does not possess any stacking faults.

Figure 3 presents a slice of the intensity along the $(31 l)$ rod. The full width of the strong fcc reflections, about 3 detector pixels, is fully resolution limited. The same instrument-limited width is found for the fcc peaks in the orthogonal direction. As illustrated in the inset, the random-stacking-induced smooth variation of the intensity along the rod closely follows the theoretical profile with the stacking probability $\alpha=0.5 \pm 0.05$. The structure factor was calculated using the theory of Wilson [3]. The absolute value of the momentum transfer vector $q(l)=(2 \pi / a) \sqrt{4\left(h^{2}+k^{2}+h k\right) / 3+3 l^{2} / 2}$ is slowly varying along the rod, where $a$ is the nearest-neighbour distance. This leads to a variation of the form factor $F(q)$, which was approximated by the form factor $F(q)=9(\sin q R-q R \cos q R)^{2} /(q R)^{6}$ of a uniform spherical particle of radius $R$ [26]. The average particle diameter $2 R=0.94 a$ is used in the calculation. The volume of the fcc crystal $V_{\text {fcc }}$ can be estimated by integrating the intensity within one reflection, while the volume of the rhcp crystal $V_{\text {rhcp }}$ is proportional to the integral along the rod within one period of the structure factor. As calculated for several periods of the structure factor between two subsequent integer values of $l$, the $V_{\text {rhcp }} / V_{\text {fcc }}$ ratio varies from $\sim 1$ to 1.5 . The variation of the obtained values of the $V_{\text {rhcp }} / V_{\mathrm{fcc}}$ ratio can be related to the effects of the dynamic (multiple) diffraction via the very strong (001) reflection [15,25]. The latter can lead to a re-redistribution of the scattered intensities between the $l$ values separated by \pm 1 . Additional effects could be induced by a possible tiny wave vector mismatch between the Bragg 
rod and the Ewald sphere. In any case, we can conclude that the rhcp and fcc crystallites have comparable size. Given the size of the irradiated sample volume $\left(150 \times 150 \times 200 \mu \mathrm{m}^{3}\right)$ and the spacing $d=a \sqrt{2 / 3}=190 \mathrm{~nm}$ between the hexagonal planes, the fcc crystal should have at least $N=400$ fcc-stacked planes without a single stacking fault.

Note that the measurements are taken about two millimeters below the liquid/solid interface such that the gravity-induced osmotic pressure is significantly higher than that at the interface for our system with large density contrast. This high crystal compression is also confirmed by the fact that no variation of the lattice constant $a$ at different heights in a crystalline part of the sample could be detected. The $6 \%$ difference between $2 R$ and $a$ can be related to several factors. Two percent can be attributed to the steric layer at the particle surface, which does not contribute to the form factor but does increase the particle size. Moreover, particle polydispersity can play a role since the positions of the minima of the form factor is mostly governed by the average particle diameter while the structure period is mainly determined by the largest spheres.

The results demonstrate that although most part of the crystalline sediment possesses an rhcp structure with the stacking probability $\alpha=0.5 \pm 0.05$, crystals with a faultless fcc structure are present as well. A similar result was observed in a different sample from the same series within the same experimental session. No indication of fcc crystallites was observed in our previous experiments $[14,15]$ with the same set of samples. Possibly, the crystals originally grow in the metastable rhcp structure and slowly transform towards the thermodynamically stable fcc phase. Our findings then clearly indicate that this transition does not process via a smooth variation of $\alpha$, but through a rearrangement mechanism via remelting as proposed in ref. [19] leading to an abrupt change in $\alpha$ from 0.5 (in rhcp) to 1 (in fcc). A similar conclusion was drawn from light powder diffraction experiments by Martelozzo et al. [18]. The latter technique, however, cannot distinguish between perfect, faultless fcc crystals and twinned crystals. The single-crystal X-ray diffraction results presented here, further strengthen the evidence for the transformation mechanism.

This conclusion seems to contradict with the results of refs. [11,13], where a pseudocontinuous spectrum of $\alpha$ was used in the data interpretation. There is a difference in the experimental approach, each of which has its own associated experimental uncertainties and possible artefacts. The studied colloidal systems may also differ in several aspects. The present study has been performed at a relatively high compression, far from the melting point [27]. The details of the interparticle interaction potential (such as "hardness/softness" of the hardcore repulsion) can play an increasingly important role with increasing pressure. Moreover, in our experiment the non-adsorbing polymer induces an effective depletion attraction between the colloidal spheres [28]. Furthermore, particle polydispersity is known to play an important role in the crystallisation dynamics [29]. The difference between colloids in terms of the polydispersity and other details of the particle size distribution can also be of importance for the structure aging, in general, and the rhcp $\rightarrow$ fcc transformation, in particular. The stacking order can be also significantly affected by an external shear field, especially at concentration close to the melting density [30]. In our crystalline sediments no flow could be observed upon tilting the capillary for a short period comparable to the measurement time. Moreover, the data were collected a few millimetres below the fluid/crystal interface, where the compression is high and therefore the effect of the shear is presumably greatly reduced.

To summarise, we have observed coexistence of rhcp and fcc crystalline phases in sediments of a mixture of hard-sphere colloids and non-adsorbing polymer. The high-resolution smallangle X-ray diffraction data suggests that the crystals have a discrete spectrum of the stacking probability: $\alpha=0.5 \pm 0.05$ in rhcp crystals and $\alpha=1$ exactly in faultless fcc crystals. The observation is interpreted as a transition from the metastable rhcp to the stable fcc 
structure via a re-melting mechanism [19]. The timescale of the rhcp $\rightarrow$ fcc transformation was theoretically predicted to be of the order of a month [19] near the melting point. One may expect that at a higher compression, as in the present experiment, such a transformation proceeds slower. Finally, from the very high sensitivity of the small-angle diffraction pattern to the crystal orientation, we conclude that the orientation of the fcc and rhcp crystals is highly correlated with a misorientation certainly smaller than about 0.1 degree [14].

$$
* * *
$$

The authors thank XINYI XIAN for the assistance during the measurements and the staff of DUBBLE beamline for the technical support. IPD acknowledges the financial support from the EU FP6-Network of Excellence Programme SoftComp for a stay in the Van 't Hoff Laboratory at the University of Utrecht. Beamtime at the DUBBLE beamline has kindly been made available by The Netherlands Organization for Scientific Research (NWO).

\section{REFERENCES}

11] Ashcroft N. W. and Mermin N. D., Solid State Physics (Saunders College, New York) 1976.

[2] Edwards O. S. and Lipson H., Proc. R. Soc. London, Ser. A, 180 (1941) 268.

[3] Wilson A. J. C., Proc. R. Soc. London, Ser. A, 180 (1941) 277.

[4] Wilson A. J. C., X-Ray Optics (Methuen \& Co. Ltd., London) 1949.

[5] Pusey P. N. and van Megen W., Nature, 320 (1986) 340.

[6] Cheng Zh. et al., Phys. Rev. Lett., 88 (2002) 015501.

[7] Vlasov Y. A., Bo X.-Z., Sturm J. C. and Norris D. J., Nature, 414 (2001) 289.

[8] Blanco A. et al., Nature, 405 (2000) 437.

[9] Bolnuis P. G., Frenkel D., Mau S.-C. and Huse D. A., Nature, 388 (1997) 235.

[10] Verhaegh N. A. M. et al., J. Chem. Phys., 102 (1995) 1416.

[11] Pusey P. N. et al., Phys. Rev. Lett., 63 (1989) 2753.

[12] Zhu J. et al., Nature, 387 (1997) 883.

[13] Kegel W. K. and Dhont J. K. G., J. Chem. Phys., 112 (2000) 3431.

[14] Petukhov A. V. et al., Phys. Rev. Lett., 88 (2002) 208301.

[15] Petukhov A. V. et al., Phys. Rev. Lett., 90 (2003) 028304.

[16] Auer S. and Frenkel D., Nature, 409 (2001) 1020.

[17] Dux Ch. and Versmold H., Phys. Rev. Lett., 78 (1997) 1811.

[18] Martelozzo V. C. et al., Phys. Rev. E, 66 (2002) 021408.

[19] Pronk S. and Frenkel D., J. Chem. Phys., 110 (1999) 4589.

[20] van Blaaderen A., Ruel R. and Wiltzius P., Nature, 385 (1997) 321.

[21] Gasser U. et al., Science, 292 (2001) 258.

[22] Verhaegh N. A. M., Asnaghi D. and Lekkerkerker H. N. W., Physica A, 264 (1999) 64.

[23] DE Hoog E. H. A. et al., Langmuir, 17 (2001) 5486.

[24] Bras W. et al., J. Appl. Crystallogr., 36 (2003) 791.

[25] Petukhov A. V. et al., Phys. Rev. E, 69 (2004) 031405.

[26] Feigin L. A. and Svergun D. I., Structure Analysis by Small-Angle X-Ray and Neutron Scattering (Plenum Press, New York) 1987.

[27] Hoover W. G. and Ree F. H., J. Chem. Phys., 49 (1968) 3609.

[28] Asakura S. and Oosawa F., J. Chem. Phys., 22 (1954) 1255; Vrij A., Pure Appl. Chem., 48 (1976) 471.

[29] Auer S. and Frenkel D., Nature, 413 (2001) 711.

[30] Ackerson B. J., J. Rheol., 34 (1990) 553; Liu J., Weitz D. A. and Ackerson B. J., Phys. Rev. E, 48 (1993) 1106; Amos R. M., Rarity J. G., Tapser P. R., Shepherd T. J. and Kitson S. C., Phys. Rev. E, 61 (2000) 2929. 